\title{
Bemerkungen zum Schutz der Grundrechte im »Raum der Freiheit, der Sicherheit und des Rechts« der Europäischen Gemeinschaft ${ }^{1}$
}

\section{Die Europäische Union im Wandel}

Als ich an der Philipps-Universität zu Marburg im Jahr 1954 begann, an einer Dissertation zu arbeiten, hätte ich nicht daran zu denken gewagt, daß ich jemals hier in der ehrwürdigen Johann Wolfgang Goethe-Universität Gelegenheit haben würde, ein paar Gedanken vortragen zu dürfen. Mein besonderer und herzlicher Dank gilt der Dekanin Frau Prof. Ogorek, der Rechtswissenschaftlichen Fakultät und Herrn Prof. Albrecht, der den Vorschlag gemacht hat, zu einem Disput über die Grundrechte und die Europäische Gemeinschaft beizutragen - ein Thema, das um so komplizierter wird, je länger man sich mit ihm beschäftigt.

1. Damals, 1954, sollte ich über die Rechtsnatur der Europäischen Verteidigungsgemeinschaft (EVG) schreiben, ob es ein Bundesstaat, ein Staatenbund oder was sonst sei - der Begriff einer »supranationalen Organisation« war damals sui generis, also eine Ausrede. Die EVG wurde nichts von alledem, weil sie in der Französischen Nationalversammlung scheiterte. Damit fanden auch meine damaligen Bemühungen ein jähes Ende. Es blieb die Frage, ob man eine europäische Nation bilden oder statt dessen ein »Europa der Vaterländer« verfassen könnte, ohne daß die mühsam erkämpfte Demokratie und die Bürger- und Menschenrechte von der wohlmeinenden intergouvernementalen Zusammenarbeit der Regierungen eingeebnet werden.

Grundrechte und Freiheiten sind nicht bloße Zugaben einer möglichst erfolgreichen Freihandelszone. Sie sind der feste Kern unserer staatlichen Existenz und die eigentlichen Grundlagen einer Europäischen Gemeinschaft, die diesen Namen verdient. Ohne die entschlossene Verwirklichung und Verteidigung der Bürgerrechte wird die Union keine solide Grundlage haben, sondern verfallen, sobald ihr ökonomischer Nutzen sinkt. Natürlich ist es leichter, ein Europa der Ministerräte und der Ministerialräte zu bauen, als ein Europa der Bürger. Und für ein Parlament ist es schwer, Autorität und Vertrauen zu erwerben, wenn die führenden politischen Kräfte der Nationen in ihm nicht vertreten sind, sondern ihm - im Gegenteil - parlamentarische Rechte nur sehr zurückhaltend zuteilen. Für die meisten Bürger Europas ist Brüssel sehr weit entfernt. Sie wollen nicht, daß über sie Macht von Beamten ausgeübt wird, die sie weder kennen, noch gewählt oder sonst legitimiert haben. Ohne aktive Beteiligung der Bürger an einer Verfassungsgesetzgebung, ohne bürgerschaftliche Legitimation wird Europa keinen Bestand haben. Darum muß es Sorge bereiten, daß die Grund- und Bürgerrechte nicht als die eigentliche Stärke unseres Gemeinwesens, sondern in zunehmendem Maße als Behinderung einer »wehrhaften Demokratie « verstanden werden, die allen möglichen »Paradigmenwechseln « geopfert werden. Dabei spielt die Zusammenarbeit der Exekutiven eine viel zu wenig beachtete Rolle.

Ursprünglich waren für die Europäischen Institutionen ökonomische Zielsetzungen beherrschend. Beim Abschluß der Verträge gab es zwar politische Absichten der un-

1 Vortrag in der Johann Wolfgang Goethe-Universität am 17. November 2006. 
terschiedlichsten Art. Zum Schutz der Menschenrechte wurden sie jedenfalls nicht geschlossen. Die staatlichen Souveränitätsrechte wurden sorgfältig gewahrt. Die Angleichung der Rechtssysteme wurde nur so weit angestrebt, wie es die wirtschaftlichen Interessen erforderten. Grundrechte fanden in den Verträgen keine Erwähnung.

Dem entspricht die Solange I-Entscheidung des Bundesverfassungsgerichts (BVerfG) vom 29. Mai 1974. ${ }^{2}$ Der Schutz der Grundrechte wurde durch das BVerfG, durch die Verbürgungen der UN-Pakte für bürgerliche und politische Rechte von 1966, die Europäische Menschenrechtskonvention (EMRK) von 1950 und durch den Europäischen Menschenrechtsgerichtshof (EGMR) wirksam gesichert.

2. Seit damals hat sich die Struktur der Verträge drastisch verändert. Beginnend mit einer »Gruppe der gegenseitigen Amtshilfe der Zollverwaltungen « am Ende der 60er Jahre und der alsbald folgenden, der Kriminalitätsbekämpfung gewidmeten sog. TREVI-Gruppe bieten die Europäischen Verträge in der heutigen Fassung von Maastricht 1993, Amsterdam 1997 mit der Aufnahme des sog. »Schengen-Besitzstandes « und mit den Vereinbarungen von Nizza 2002 ein völlig anderes Bild.

Die geradezu beschwörende und ständig wiederholte Bezeichnung »Raum der Freiheit, der Sicherheit und des Rechts $\ll^{3}$ markiert einen Wandel, mit dessen Intensität weder die Sicherung der Grundrechte, noch die demokratische Legitimation der EU-Entscheidungen Schritt gehalten haben. Nach heutigem Verständnis des Vertragsrechts der Union kann die Bundesregierung völkervertragsrechtlich verbindliche, grundrechtsrelevante Verpflichtungen eingehen, ohne daß es dazu einer Mitentscheidung des Europäischen Parlamentes oder des Bundestages bedarf. Auch der vom Bundestag bereits verabschiedete Entwurf eines Europäischen Verfassungsvertrages (EVerfVE) enthält erstaunliche, in der Öffentlichkeit unbeachtet gebliebene Regelungen, die den Kern des Grundgesetzes berühren. So könnte die Bundesregierung nach der Solidaritätsklausel des Art. I - 43 EVerfVE auf der Grundlage eines Ratsbeschlusses auch im Inland eigene militärische Mittel und Soldaten anderer EU-Mitglieder zur Abwehr einer terroristischen Bedrohung anfordern bzw. einsetzen, nach Art. III - 329 EVerfVE ohne jede parlamentarische Mitentscheidung. Das ist angesichts der Entscheidung des BVerfG zum Luftsicherheitsgesetz vom 15. Februar 2006 eine für das Grundgesetz erstaunliche Regelung. Der Europäische Rat hat sie schon am 25. März 2004 »politisch in Kraft gesetzt«, was immer das heißen möge, ohne daß der Bundestag das auch nur mit einem Wort erörtert hätte. ${ }^{4}$

2 Vgl. BVerfG NJW 1974, $1697 \mathrm{ff}$.

3 Vgl. zu diesem Begriff Denninger, Freiheit durch Sicherheit?, in Koch (Hrsg), Terrorismus - Rechtsfragen der äußeren und inneren Sicherheit, 2000, S. $83 \mathrm{ff}$.

4 Der Bundespräsident hat das RatifizierungsG zum EVerfVE wegen einer am BVerfG anhängigen VerfB noch nicht unterzeichnet.

Die Erklärung 9 zur Schlußakte, die Solidaritätsklausel ziele nicht darauf ab, das Recht eines Mitgliedsstaates zu beeinträchtigen, das geeignetste Mittel zur Erfüllung seiner Solidaritätspflicht zu treffen, ändert nichts. Der Verfassungsvertrag erlaubt jedenfalls, daß im Inland auch militärische Mittel - also Soldaten und Kriegswaffen - eingesetzt werden können, wenn der Europäische Rat das beschließt. Vgl. dazu Schmidt-Radefeldt, UBWV 2006, 161 ff.: die Bundesrepublik komme in eine merkwürdige Lage, wenn sie auf dieser Grundlage die Bundeswehr in Frankreich in einer Weise einsetzen wollte, die der Bundeswehr im Inland nach dem Grundgesetz nicht erlaubt sei. Die Solidaritätsklausel mache deutlich, »daß auch die innere Sicherheit eines Mitgliedsstaates nicht mehr allein eine nationale Angelegenheit darstellt, sondern längst zu einer europäischen Frage geworden ist, die letztlich eine europäische Antwort sucht.«, vgl. a.a.O., S. 167. 
Der EVerfVE enthält zwar in seinem Teil II eine - auch wegen der Berücksichtigung sozialer Rechte - beachtenswerte Grundrechtscharta. Aber gleichzeitig strotzt der EVerfVE in seinem III. Teil über die justitielle und polizeiliche Zusammenarbeit von einer solchen Vielfalt unbestimmter Begriffe, mit Geldbußen bewehrter Verpflichtungen, von Leitlinien und verpflichtenden Zielen, daß von einer Bestimmtheit und Voraussehbarkeit des Umfangs der Übertragung von Hoheitsrechten, die Art. 23 Abs. 1 GG nach ständiger Rechtsprechung des BVerfG fordert, ${ }^{5}$ keine Rede mehr sein kann. Was auf der einen Seite den Abschluß des Verfassungsvertrages erleichterte, ruinierte auf der anderen Seite seine Verständlichkeit und damit auch seine Akzeptanz.

Man kann mit großer Sicherheit sagen, daß dieser Vertrag bei einer Volksabstimmung in den meisten Mitgliedsländern durchgefallen wäre und daß er genau deswegen auch in der Bundesrepublik keiner Volksabstimmung unterworfen wurde.

3. Wenn durch Beschlüsse oder Handlungen europäischer Institutionen Grundrechte berührt werden sollen, dann setzt das zunächst voraus, daß diesen Institutionen Hoheitsrechte nach Art. 23 Abs. 1 GG wirksam übertragen wurden. Dazu hat das BVerfG in ständiger Rechtsprechung ${ }^{6}$ fünf Grundsätze entwickelt, die weitgehend unstreitig sind - wenn man die Frage ausklammert, wer zuletzt darüber entscheidet, ob diese Voraussetzungen erfüllt sind: das BVerfG oder der Europäische Gerichtshof (EuGH). Diese wenigen Grundsätze kann man so zusammenfassen:

a. Art. 23 GG läßt die Übertragung von Hoheitsrechten zur Bildung einer rechtsstaatlichen und demokratischen Europäischen Union auch dann zu, wenn sie nur einen dem Grundgesetz im wesentlichen vergleichbaren Rechtsschutz gewährleistet.

b. Die in Art. 1 und 20 GG niedergelegten Grundsätze sind gemäß Art. 23, 79 Abs. 3 GG unantastbar. Dazu gehört auch das Erfordernis der demokratischen Legitimation europäischer Entscheidungen gemäß Art. 20, 38 GG.

c. Die nationale Identität des Mitgliedsstaates muß gewahrt bleiben, tragende Grundsätze des Verfassungsrechtes dürfen nicht abgebaut werden.

d. Die Übertragung der Hoheitsrechte muß dem Bestimmtheitsgebot entsprechen. Es muß für den Gesetzgeber voraussehbar sein, welche Befugnisse er verliert und der Europäischen Gemeinschaft überträgt.

e. Europäische Entscheidungen, die die übertragenen Rechte überschreiten, können keine innerstaatlichen Rechtswirkungen auslösen. Auch die richterliche Rechtsfortbildung durch den EuGH muß diese Grenze beachten.

Es bleibt allerdings der kritische Punkt übrig, wer diese Entscheidungen im Konfliktfall letztinstanzlich treffen kann und muß. Während das BVerfG diese Frage tendenziell offen halten will, bemüht der EuGH sich darum, den Vorrang des Gemeinschaftsrechts und die Letztverbindlichkeit der eigenen Entscheidungen sogar in der

5 Vgl. die Maastricht-Entscheidung, NJW 1993, $3047 \mathrm{ff}$.

6 Vgl. insbes. die Entscheidungen »Solange II«, NJW 1987, 577 ff.; zum Vertrag von Maastricht, NJW 1993, 3047 ff.; »Bananenmarkt«, NJW 2000, 3124 ff. und die Entscheidung zum Europäischen HaftbefehlsG, NJW 2005, 2289 ff. Vgl. dazu zusammenfassend Günter Hirsch, EuGH und BVerfG - Kooperation oder Konfrontation?, NJW 1996, 2457 ff. u.d.d. Zit. 
Frage durchzusetzen, ${ }^{7}$ ob eine seiner Entscheidungen auf zulässiger richterlicher Rechtsfortbildung beruht oder unzulässig ultra vires ergeht.

4. Das BVerfG geht davon aus, daß die Voraussetzungen für eine Übertragung von Hoheitsrechten bei den gegenwärtigen Vertragswerken erfüllt sind. Auch wenn es für sich im Prinzip auch weiterhin in Anspruch nimmt, den Grundrechtsschutz i n der Bundesrepublik, also auch gegenüber europäischen Rechtsakten zu überwachen, so hat es doch seit der Solange II-Entscheidung die Auffassung vertreten, daß ein ausreichender Grundrechtsschutz i. S. d. Art. 23 GG durch den EuGH gegeben und die Anrufung des BVerfG in den zu entscheidenden konkreten Fällen nicht nur unbegründet, sondern unzulässig sei.

Es stellt sich aber die Frage, ob diese Rechtsprechung im Interesse eines wirksamen innerstaatlichen Rechtsschutzes angesichts der Dynamik weiterentwickelt werden muß, in der der sog. »Raum der Freiheit, des Rechts und der Sicherheit« zu einem staatlichen Surrogat ausgebaut wird.

Auf der einen Seite wird zwar in Art. 6 Abs. 3 EUV die Erhaltung der staatliche Identität betont und erklärt, daß die sog. »Dritte Säule« - der justitielle und polizeiliche Bereich des Vertrages - zur »intergouvernementalen Zusammenarbeit«, also nicht zur eigentlichen Union gehört und nur völkervertragsrechtliche Bindungen ermöglicht. ${ }^{8}$ Auf der anderen Seite wird dem Rat in Art. 67 EGV die Einführung von Mehrheitsentscheidungen nach 5 Jahren ohne Mitentscheidung der Parlamente in Bereichen der klassischen Innenpolitik - Asyl, Einwanderung, Visa - anheimgestellt, werden die Rahmenbeschlüsse gemäß Art. 34 EUV der rechtlichen Wirkung von Richtlinien gemäß Art. 249 Abs. 3 EGV angenähert und der Rechtsprechung des EuGH im Wege von Vorentscheidungen unterworfen.

Vor allem aber wird in dem sog. Haager Programm eine Zusammenarbeit entwickelt, die das innerstaatliche Recht der Mitgliedsstaaten auch da erfassen soll, wo es mit der grenzüberschreitenden Zusammenarbeit innerhalb der EU oder der EG nichts mehr zu tun hat. Darauf hat das BVerfG in seiner Rechtsprechung bisher nicht reagiert.

\section{Raum der Freiheit und der Sicherheit}

Die Begriffe Freiheit und Sicherheit werden in den Verträgen mit großer Schlichtheit nebeneinander gestellt.

Es ist richtig, daß es Freiheit ohne Sicherheit nicht gibt. Aber es ist falsch, daß immer mehr Sicherheit auch immer mehr Freiheit bedeuten würde. Sicherheit und Freiheit sind politische Zielsetzungen, von denen die Forderung nach Sicherheit im nationalen wie im europäischen Bereich in dramatischer und beklagenswerter Weise dominant geworden ist, was Art. 29 EUV überflüssiger Weise ausdrücklich bestätigt.

1. Die Bundesrepublik hat in den letzten 30 Jahren eine innenpolitische Aufrüstung ohnegleichen vollzogen. Sie sollte vor allen denkbaren kriminellen Gefahren schützen

7 Vgl. Günther Hirsch a.a.O., S. 2462 ff.

8 BT-Entschließung v. 5.3.1998, BT-Drs.13/9913 v. 13.2.1998, S. 1 ff. 
und hat eine erhebliche Demontage unserer Rechtstraditionen bewirkt. Höhere Strafdrohungen, außerordentliche Ausdehnung der Telefon-, Post-, und sonstigen Telekommunikationsüberwachungen, erleichterte Haftgründe, bis zur Verfassungswidrigkeit ausgedehnte präventiv-polizeiliche Eingriffsrechte, die Speicherung von Daten über Personen, von denen man erst in der Zukunft Straftaten erwartet, die Einführung nachrichtendienstlicher Mittel in die Strafprozeßordnung, elektronisches Belauschen in der Öffentlichkeit und im privaten Bereich - das sind nur Beispiele, die überdies fast sämtlich ohne empirische und nachvollziehbare Erfolgskontrolle geblieben sind. Mahnten Richter des BVerfG noch in einer Entscheidung von 1970, es gelte, den Anfängen zu wehren, so hieß es schon in der Entscheidung zum »Großen Lauschangriff« von 2004, es gelte, nicht den Anfängen, sondern dem bitteren Ende zu wehren. ${ }^{9}$ Dem »Großen Lauschangriff « und dem Attentat vom 11. September 2001 folgten »Schily I und II « und das Luftsicherheitsgesetz überwiegend im parlamentarischen Blindflug. ${ }^{10}$ Die Reihe setzt sich mit den Gesetzentwürfen zu einer gemeinsamen Datei der Sicherheitsbehörden, zur Ergänzung des Terrorismusbekämpfungsgesetzes und voraussichtlich zur Vorratsdatenspeicherung fort.

2. In der EU gibt es nach dem 11. September 2001 einen ganz massiven Aufbau sicherheitsrelevanter Bestrebungen strafrechtlicher und strafverfahrensrechtlicher Art, und zwar auf der Grundlage der sog. »Dritten Säule«. Es genügt vollkommen, sich den Rahmenbeschluß des Rates zum Europäischen Haftbefehl vom 13. Juni 2002, den Rahmenbeschluß zur Terrorismusbekämpfung vom gleichen Tage, die Verordnung vom 16. Dezember 2003 zur Sicherung der Zivilluftfahrt und schließlich das höchst bemerkenswerte Haager Programm des Rates zur Stärkung von Freiheit, Sicherheit und Recht in der Europäischen Union anzusehen. ${ }^{11}$

Das Programm soll dazu führen, daß $»$ Freiheit, Recht, Sicherung der Außengrenzen, innere Sicherheit und Verhütung des Terrorismus (...) als ein für die Union insgesamt unteilbares Ganzes betrachtet werden.« Es sei ein »multidisziplinäres und abgestimmtes Vorgehen sowohl auf der Ebene der EU als auch auf der Ebene der Mitgliedstaaten zwischen den zuständigen Strafverfolgungsbehörden, insbesondere Polizei, Zoll und Grenzschutz erforderlich. ${ }^{12}$

Es enthält ein ganzes Regierungsprogramm in den Bereichen des Asyl- und Einwanderungsrechtes, der Nutzung biometrischer Merkmale und der Informationssysteme, der Integrationspolitik, der allgemeinen und besonderen polizeilichen $\mathrm{Zu}$ sammenarbeit, des völlig uneingeschränkten grenzüberschreitenden Datenaustauschs im online-Verfahren, der Einführung des Europäischen Haftbefehls, der gegenseitigen

9 Vgl. das Minderheitenvotum der Richter Geller, v. Schlabrendorff und Rupp in dem Urteil v. 15.11.1970 zu Art. 10 GG, BVerfGE 30, 1 ff., 40, und das Minderheitenvotum der Richterinnen Jaeger und Hohmann-Dennhardt im Urteil zum »Großen Lauschangriff« v. 3.3.2004, NJW 2004, 999 ff.

10 Vgl. dazu die eindringliche Darstellung von Düx, Globale Sicherheitsgesetze und weltweite Erosion von Grundrechten, ZRP 2003, $189 \mathrm{ff}$.

11 Vgl. Amtsbl. 2002, L 190/1; Amtsbl. 2002 L 164/3; Amtsbl. 2002, L 355/1; Amtsbl. 2005, C 53/1.

12 Vgl. a.a.O., C 53/2. 
Anerkennung von Entscheidungen, der Angleichung von Straftatbeständen, der Harmonisierung von Beschuldigtenrechten und Beweiserhebungsregeln und schließlich über den Ausbau von Europol - über deren völlig überholte und antiquierte strafrechtliche Immunität kein Wort verloren wird, ebensowenig wie darüber, daß der EuGH für die Kontrolle von Europol-Maßnahmen nur sehr eingeschränkt zuständig ist und Teile der Europol-Konvention mit demokratischen Prinzipien wenig zu tun haben. $^{13}$

Grundsätzlich neu am Haager Programm sind das Anerkennungs- und das Verfügbarkeitsprinzip.

Während das Verfügbarkeitsprinzip auf einen einheitlichen Zugang zu sicherheitsbehördlich gespeicherten Datenbeständen der Mitgliedsstaaten abzielt und damit einheitliche Regeln zu einem europäischen Datenstatut der Sicherheitsbehörden erfordert -, greift das Anerkennungsprinzip noch weiter.

Durch die grundsätzliche Anerkennung der Rechtswirksamkeit polizeilicher oder justitieller Zwangsmaßnahmen eines Mitgliedsstaates in allen anderen Unionsstaaten soll der jeweilige Vollstreckungsstaat seine sicherheitsrechtlichen Instrumente zugunsten des Verfolgerstaates ohne Rücksicht darauf einsetzen, ob der anerkannte Rechtsakt mit der eigenen Rechtsordnung im Einklang steht, also z. B. ob das in Frage stehende Verhalten eines Bürgers im Vollstreckungsstaat überhaupt strafbar ist. Es ist offenkundig, daß auf diese Weise ein immanenter Zwang zur Einführung von gemeinsamen rechtlichen Mindeststandards auch auf Gebieten entsteht, die mit wirtschaftsrelevanten Wettbewerbsbedingungen nichts zu tun haben. So sind Rahmenbeschlüsse über den Haftbefehl, über Informationssysteme, Kinderpornographie, Bestechung im privaten Bereich, über Geldstrafen und -bußen, über die Einziehung von Erträgen aus Straftaten und Tatwerkzeugen, über die Sicherstellung von Beweismitteln und Vermögensgegenständen, über Beweisanordnungen zur Erlangung von Sachen, Schriftstücken und Daten zur Verwendung im Strafverfahren ergangen, die nach Art. 31, 31a, 34 EUV allenfalls im Bereich der Organisierten Kriminalität, des Terrorismus und des illegalen Drogenhandels eine Rechtsgrundlage haben könnten. Dabei werden auch im Grundgesetz verankerte Verfahrensgarantien und die Rechte von Berufsgeheimnisträgern ohne parlamentarische Entscheidung mit leichter Hand ausgehebelt. $^{14}$

Diese Regelungen erfassen auch Sachverhalte ohne Kooperationserfordernisse, beziehen sich also auf rein innerstaatliche Bereiche der Strafverfolgung und

13 Vgl. Streinz, EUV/EGV, Kommentar, Rdnr. 15 zu Art. 35 EUV. Der EuGH kann nur Vorabentscheidungen über die Auslegung der Europol-Konvention treffen. Vgl. zur Konvention Albrecht, Simitis u.a., 11 Thesen zur Entwicklung rechtsstaatlicher Grundlagen im europäischen Strafrecht, insbes. zu Art. 8 (Verdacht) und 10 (polizeiliche Datenverarbeitung) EPK, KritV 2001, 279 ff.; sowie Albrecht/Braum mit äußerst kritischer Bewertung des »Europ. Strafrechts«, KritV 2001, 312 ff., 348.

14 Vgl. Gazeas, Die Europäische Beweisanordnung - Ein weiterer Schritt in die falsche Richtung?, ZRP 2005, 18 ff.; Kaiafa, Bemerkungen zur rechtsstaatlichen Entwicklung des Europ. Strafrechts, KritV 2001, 290 ff., 295. 
Gefahrenabwehr. ${ }^{15}$ Es ist paradox, daß auf diese Weise die zur intergouvernementalen Zusammenarbeit gehörende »Dritte Säule« zum eigentlichen Instrument einer inhaltlichen, über Sachverhalte der Zusammenarbeit weit hinausgehenden Integration geworden ist.

3. Diese Zielsetzung sprengt die dem Vertrag zugrunde liegende Struktur der »Dritten Säule« als Völkervertragsrecht. Es geht nicht mehr um völkerrechtliche Vereinbarungen, deren Erfüllung sanktionslos unterlassen werden kann, sondern um Beschlüsse, die ausdrücklich als Teil des bestehenden supranationalen Vertragswerks verstanden werden. Sie werden von Organen der Union gefaßt und sollen natürlich innerstaatliche Wirkung erlangen. Es war voraussehbar, daß der EuGH seine Rechtsprechung über die unmittelbare Geltung von Richtlinien alsbald auch auf die ohne parlamentarische Entscheidung ergehenden Rahmenbeschlüsse der »Dritten Säule« übertragen würde. ${ }^{16}$

Die europäische Exekutive will, natürlich in bester Absicht, ein höchst effektives Gebilde aufbauen und sich dabei nicht zu lange mit der theoretischen und politisch lästigen Frage aufhalten, ob es sich dabei im Ergebnis schon um einen Staat handelt oder vielleicht noch nicht.

4. In der deutschen Öffentlichkeit haben nur wenige Details des Haager Programms eine Rolle gespielt, so der vom Bundestag erzwungene Gesetzesvorbehalt in »Schily II « über die Aufnahme biometrischer Merkmale in die Pässe, der kurzerhand durch eine Verordnung des Rates gegenstandslos gemacht wurde, ${ }^{17}$ der Europäische Haftbefehl mit der wichtigen Entscheidung des $\mathrm{BVerfG}^{18}$ und die noch nicht abgeschlossene Diskussion über die sog. Vorratsdatenspeicherung, die einen politisch abenteuerlichen Weg genommen hat. ${ }^{19}$

15 Vgl. Hecker, Die Europäisierung der inneren Sicherheit, DÖV 2006, 273 ff., 279, 280. Die Kommission bereitet sogar Rahmenbeschlüsse zur Unschuldsvermutung und $\mathrm{zu}$ den Beschuldigtenrechten im Strafverfahren vor, wohl im Vorgriff auf Art. III - 270 Abs. 2 EVerfVE, vgl. Hecker a.a.O., S. 280. Vgl. auch Albrecht, Simitis u.a., a.a.O. Während die bisherigen Verträge keine Rechtsgrundlage für die Einführung eines europäischen Strafrechts enthalten, soll das zukünftig in den EVerfVE aufgenommen werden, Art. III - 271 EVerfVE.

16 Vgl. EuGH Vorlageentscheidung v. 16.6.2005 (Rs. C - 105/3 - Pupino), NJW 2005, 2839 ff.; EuGH v. 11.7.2006 »ELOG«, NJW 2006, 2465. Das BVerfG hätte durchaus Veranlassung gehabt, sich gerade bei der Haftbefehlsentscheidung in den Urteilsgründen näher mit der Frage auseinanderzusetzen, welche Einflußmöglichkeiten die beteiligten Parlamente gehabt hatten. Ursprünglich sollte das Haftbefehlsverfahren durch zwei Übereinkommen nach Art. 34 Abs. 2 d EUV - früher Art. K 3 - eingeführt werden. Das scheiterte aber daran, daß Frankreich und Italien die Übereinkommen nicht ratifizierten. Daraufhin wurde der Weg über den Rahmenbeschluß gewählt, um die Parlamente auf diese Weise zu zwingen, die Entscheidungen zu treffen, die sie freiwillig nicht hatten treffen wollen. Vgl. Schilling, Gesetz und Demokratieprinzip. Eine Inkohärenz der Rechtsprechung des EGMR?, Archiv d. Völkerrechts, 2006 (44), 72.

17 Vgl. VO Nr. 2252/2004 v. 13.12.2004, Amtsbl. 2004, L 385/1.

18 Vgl. Beschluß v. 18.7.2005, NJW 2005, 2289 ff.; Kaleck, Der Europäische Haftbefehl Ein Schritt zu früh in den gemeinsamen Strafprozeß?, in Müller-Heidelberg (Hrsg) Grundrechte - Report 2003, S.142 ff.; Leutheusser-Schnarrenberger, Der Europäische Haftbefehl, in Roggan (Hrsg) Mit Recht für Menschenwürde und Verfassungsstaat, BWV 2006, 103 ff. 
Natürlich muß und wird ein entsprechendes nationales Gesetz vor das BVerfG gebracht werden, weil hier zum ersten Mal Private verpflichtet werden sollen, für polizeiliche Zwecke Daten, die dem Schutz des Art. 10 GG unterliegen, zu erheben und zu speichern, ohne sie selbst zu benötigen und ohne daß hinsichtlich der Betroffenen irgendeine konkrete polizeiliche Veranlassung besteht. Das verstößt nach meiner Überzeugung gegen die Art. 1 und 2 GG, gegen die Menschenwürde ebenso wie gegen das informationelle Selbstbestimmungsrecht.

5. Insgesamt ist inzwischen ein kaum noch überschaubares Geflecht von Beschlüssen, Protokollnotizen, Gremien und Eingriffsrechten entstanden. Das hat schon zu dem Ruf geführt, sich vom traditionellen Staatsbegriff zu verabschieden. Die Union stößt an ihre konzeptionellen Grenzen. ${ }^{20}$

Wenn man es den Exekutiven überläßt, die justitielle und polizeiliche Zusammenarbeit zu organisieren, anstatt in eine exakte europäischen Gesetzgebung einzutreten, dann werden sie das mit denselben Mitteln unternehmen, die bei den wirtschaftlichen Zielsetzungen der Union erfolgreich waren. Es geht dann um die Anerkennung der jeweils bereits getroffenen Entscheidungen, des jeweils schärfsten Strafrechts, eine Nivellierung der Bürgerrechte auf dem jeweils niedrigsten Niveau, ${ }^{21}$ um die Perfektionierung der administrativen Zusammenarbeit und die größtmögliche Effektivität und nicht darum, Freiheiten nicht mehr einzuschränken, als unbedingt erforderlich. ${ }^{22}$

Die Entscheidungsfreiheit der nationalen Parlamente und ihre Eigenverantwortung wird dabei zur politischen Fiktion. Sie machen sich von den Ermessensspielräumen abhängig, die ihnen die europäischen Vorgaben gerade noch lassen. Der in der Sache »Francovich « zuständige Generalanwalt hat das in seinen Schlußanträgen sehr schön beschrieben: »Ich halte die Behauptung nicht für übertrieben, daß sich der Gesetzgeber (eines Mitgliedstaates), wenn es um die Umsetzung einer Richtlinie (der Gemeinschaft) geht, in einer ähnlichen Lage befindet wie die Verwaltung, die ein Gesetz durchzuführen hat. « ${ }^{23}$

Materiell übernimmt die Exekutive die Rolle des eigentlichen Gesetzgebers. Sie ist zwar nach den Zusammenarbeitsgesetzen zur Information des Bundestages und natür-

19 Die Speicherung wurde zunächst als Rahmenbeschluß geplant, dann wegen fehlender Einstimmigkeit im Rat und zur Besänftigung des Europäischen Parlamentes im März 2006 als Richtlinie nach Art. 249 EGV, vgl. RL 2006/24/EG, Amtsbl. 2006, S. 97 ff., beschlossen. Irland hat wegen dieser Rechtsgrundlage vor dem EuGH Klage erhoben, über die noch nicht entschieden ist.

20 Vgl. Leutheusser-Schnarrenberger, Ein System gerät außer Kontrolle: Das Schengener Informationssystem, ZRP 2004, 97 ff. Vgl. die Auflistung bei Baldus, Polizeiliche Zusammenarbeit im Raum der Freiheit, der Sicherheit und des Rechts, in Bergmann (Hrsg), Recht und Politik der Europäischen Union. Der Integrationsverbund vor der Osterweiterung, S. 35 ff. Baldus plädiert im Ergebnis für eine Auflösung des traditionellen Staatsbegriffs, weil der nationale Staat nicht mehr in der Lage sei, seine Sicherheitsaufgaben ohne internationale polizeiliche Zusammenarbeit zu erfüllen. Vgl. Hecker, a.a.O., S. 280.

21 Vgl. insbes. Schünemann, Europ. HaftB und EU-VerfassungsE auf schiefer Ebene, ZRP 2003, $185 \mathrm{ff}$.

22 Vgl. Albrecht, Die Normgenese des EurHaftBG, in Roggan (Hrsg), Mit Recht für Menschenwürde und Verfassungsstaat, S. 115, 123 ff.; Hecker, a.a.O. S. 275.

23 Vgl. Generalanwalt Mischo, Schlußantrag Nr. 47, EuGRZ 1992, 62; Schilling, Allgemeine Rechtsgrundsätze des EG-Rechts, EuGRZ 2000, 1 ff., 43. 
lich des Bundesrates verpflichtet. Sie hat deren Stellungnahmen zu »berücksichtigen«, aber die bleiben letzten Endes unverbindlich. ${ }^{24}$ Hinzu kommt, daß die Fachminister der Bundesregierung nicht vom Vertrauen des Bundestages abhängen. Die vom BVerfG in der Maastricht-Entscheidung erwähnte Möglichkeit des Kanzlersturzes ${ }^{25}$ ist jedenfalls politisch keine ernsthafte Einflußmöglichkeit auf Entscheidungen der Ministerräte und begründet keine tatsächliche politische Legitimationskette.

Dieses eklatante politische Demokratiedefizit steht in einem wachsenden Spannungsverhältnis zu der wachsenden Integration im polizeilichen und justitiellen Raum. Um so dringender wird die Frage, ob und in welchem Umfang die Gerichtsbarkeit angesichts der diese Entwicklung offenbar hinnehmenden Parlamente beitragen kann, die Grundrechte und demokratischen Grundstrukturen zumindest in der Bundesrepublik zu schützen.

\section{Raum des Rechts}

Wer in dem Raum der Sicherheit den Raum des Rechts sucht, sieht sich einer verwirrenden Kooperationsbereitschaft der beteiligten obersten Gerichte gegenüber. Neben drei obersten Gerichten - BVerfG, EuGH und EGMR - hat man es mit drei kodifizierten Grundrechtsordnungen zu tun, der Europäische Menschenrechtskonvention, dem Grundgesetz und der Grundrechtscharta der Union, die bisher nicht in Kraft getreten ist und einem weiteren Grundrechtskatalog, der vom EuGH als Richterrecht entwickelt wurde. Das sollte reichen, ist aber eher verwirrend.

1. Die Anrufung des EGMR ist seit dem 11. Protokoll auch durch eine individuelle Klage möglich. ${ }^{26}$ Die EMRK gilt in der Bundesrepublik als Bundesgesetz. Das BVerfG läßt eine Verfassungsbeschwerde wegen Verletzung der EMRK zu und verlangt von den Fachgerichten die grundsätzliche Beachtung der Konvention und der Entscheidungen des EGMR als Teile des Bundesrechtes. ${ }^{27}$

Die Entscheidungen des EGMR haben wesentliche Folgen für Gesetzgebung und Verwaltung gehabt. ${ }^{28}$ Ernsthafte rechtliche Komplikationen sind weder bisher aufgetreten noch zukünftig zu erwarten.

2. Komplizierter ist das Verhältnis des EGMR zum Unionsrecht, weil die Union und die Europäische Gemeinschaft keine Mitglieder der EMRK sind. Der EGMR prüft das Recht der EU/EG aber indirekt, indem er von den Mitgliedstaaten der Konvention verlangt, die Rechte der EMRK auch gegenüber der Union durchzusetzen. ${ }^{29}$ Der EuGH

24 Ganz zu schweigen davon, daß der Bundestag von seiner Mitwirkungsmöglichkeit bisher kaum Gebrauch gemacht hat, vgl. Gazeas, a.a.O., S. 20.

25 Vgl. BVerfG, a.a.O., S. 3053.

26 Die mangelhafte organisatorische und finanzielle Ausstattung des EGMR hat gegenwärtig zu einer Belastung des Gerichts mit 80.000 unerledigten Fällen geführt.

27 Vgl. insbes. den sorgfältig begründeten Beschluß v. 14.10.2004 in der Sache Görgülü, NJW 2004, $3407 \mathrm{ff}$.

28 Vgl. die sehr positive Würdigung bei Papier, Umsetzung und Wirkung der Entscheidungen des EGMR aus der Perspektive der deutschen nationalen Gerichte, EuGRZ 2006, $1 \mathrm{ff}$.

29 Vgl. Entscheidung v. 18.2.1999 (Matthews), EuGRZ 1999, 200. 
hat seinerseits Differenzen zur Rechtsprechung des EGMR im allgemeinen vermieden und die Auslegungsregeln des EGMR zu den Konventionsrechten übernommen. ${ }^{30}$

Eine Kollision zwischen EU/EG-Recht und der EMRK ist allerdings dann möglich, wenn eine Entscheidung der Union ein Recht der EMRK einschränken würde. So könnte z. B. ein Rahmenbeschluß nicht als das nach der EMRK für Grundrechtsbeschränkungen geforderte gesetzgeberische Verfahren gelten. ${ }^{31}$

Sollte die Grundrechtscharta des EVerfVE unverändert in Kraft treten, dann werden die Unterschiede zwischen der Charta und dem Katalog der EMRK eine wachsende Rolle spielen. Der Kernpunkt liegt darin, daß die Vorbehalte für Einschränkungen der Grund- und Menschenrechte völlig unterschiedlich geregelt sind. In der EMRK sind sie für jedes einzelne Menschenrecht spezifisch geregelt, dagegen in Art. II - 112 EVerfVE für alle Grundrechte der Charta gemeinsam als allgemeiner und außerordentlich unpräziser Vorbehalt formuliert worden.

3. Weit komplizierter, schwerer erkennbar und im Ergebnis unbefriedigend ist das Verhältnis zwischen dem EuGH und dem BVerfG.

Der EuGH hat die Europäische Gemeinschaft durch seine Rechtsprechung konsequent ausgebaut. Er hat - nach dem massiven Anstoß durch die Solange I-Entscheidung des BVerfG - die im Vertrag fehlenden Grundrechte durch Richterrecht eingeführt, die Pflicht zur Anwendung der Richtlinien durch a 11 e Träger der öffentlichen Verwaltung und in bestimmten Grenzen die Pflicht zur Beachtung auch der einzelstaatlich noch nicht umgesetzten Richtlinien eingeführt und sogar für Rahmenbeschlüsse behauptet. ${ }^{32}$ Er ist der Auffassung, daß die nationalen Parlamente bei der Umsetzung der Richtlinien in nationales Recht nur an die Europäischen Grundrechte gebunden seien und die Grundrechte der eigenen Verfassungen dabei keine Rolle mehr spielen. ${ }^{33}$

Seine Rechtsprechung zu den Grundrechten ist allerdings nicht ohne Kritik geblieben, weil er der Effektivität von Verwaltungsentscheidungen für den Gemeinsamen Markt stets einen deutlichen, entscheidenden Vorrang gegenüber dem individuellen

30 Vgl. Dörr, a.a.O., S. 1088 ff., 1090 und die dortige Darstellung einzelner Differenzen in der Rspr. von BVerfG, EGMR und EuGH, a.a.O., S. $1093 \mathrm{ff}$.

31 Vgl. Schilling, a.a.O., S. 57 ff., der zutreffend darstellt, daß ohne eine solche parlamentarische Mindestanforderung der Gesetzesbegriff der EMRK nicht erfüllt ist.

32 Vgl. EuGH, Slg. 1989, 1839 - Constanzo, vgl. dazu ausführlich Jarass, Folgen der innerstaatlichen Wirkung von EG-Richtlinien, NJW 1991, 2665 ff. Vgl. zur Einführung von Schadensersatzansprüchen bei verspäteter Umsetzung einer Richtlinie die Francovich-Entscheidung des EuGH, NJW 1992, 165 und zu den Rahmenbeschlüssen die Pupino-Entscheidung EuGH v. 16.6.05, NJW 2005, 2839. Vgl. ferner Schwarze, Grundzüge und neuere Entwicklungen des Rechtsschutzes im Recht der EG, NJW 1992, 1065 ff. Dem EuGH ist in diesem Zusammenhang vorgehalten worden, er praktiziere seine Funktion zunehmend in Form einer Institution, die anstelle der nationalen Regierungen durch »Kompetenzkreation « einen Souveränitätsverlust der Mitgliedstaaten zugunsten der EG bewirke, vgl. im einzelnen Scholz/Hofmann, Perspektiven der europäischen Rechtsordnung, ZRP 1998, 295 ff., mit der Überlegung, daß es »nötigenfalls« einer Kontrolle des EuGH auch durch das BVerfG bedarf, das mit höherer demokratischer Legitimation ausgestattet sei, a.a.O., S. 302.

33 Thym, Europäischer Grundrechtsschutz, NJW 2006, 3249 ff. 
Rechtsschutz gegeben hat ${ }^{34}$ und weil die Verträge keine Verfassungsbeschwerde kennen, sondern den individuellen Zugang zum Gericht gemäß Art. 230 Abs. 4 EGV und Art. 35 EUV, abgesehen von wenigen Ausnahmen, auf das Vorlageverfahren beschränken. ${ }^{35}$ Daran hat der EuGH selbst durchaus berechtigte Zweifel geäußert. Es sei »fraglich, ob die Nichtigkeitsklage, die für den Einzelnen nur im Hinblick auf Rechtsakte eröffnet ist, die ihn unmittelbar und individuell betreffen, ausreicht, um ihm einen effektiven Rechtsschutz gegen Verletzungen seiner Grundrechte zu gewähren, die sich aus der Gesetzgebungstätigkeit der Organe ergeben können. « ${ }^{36}$

Der in Nizza feierlich verkündete Grundrechtskatalog wird keine deutliche Verbesserung dieser Lage mit sich bringen. Die Charta wird zwar auch ohne formelle Rechtskraft als gemeinsamer Grundstock der Verfassungsrechte der EU-Mitglieder i.S.d. Art. 6 EUV in der Rechtsprechung des EuGH beachtet werden. ${ }^{37}$ Aber sie eröffnet keine unmittelbare Rechtsbeschwerde und der für den Bestand der Rechte entscheidende generelle Einschränkungsvorbehalt des Art. II - 112 EVerfVE ist im Gegensatz zu den strikten Regelungen der EMRK so unklar, daß die konkreten Abwägungsmaßstäbe zwischen individueller Freiheit und genereller Effektivität nicht vorhersehbar sind. ${ }^{38}$

4. Das Bundesverfassungsgericht hat mit »Solange II «, dem Maastricht-Urteil, der Bananenmarkt- und der Haftbefehls-Entscheidung ${ }^{39}$ im wesentlichen nur verfahrensrechtlich entschieden und offen gelassen, ob das EU-Recht dem Grundgesetz übergeordnet ist oder ob das Gericht auch Rechtsakte der Gemeinschaft in der Bundesrepublik dem Grundgesetz unterwerfen will. Das BVerfG erkennt zwar den vom EuGH kraft Richterrechts geschaffenen Grundrechtsschutzes an, aber eben nur,

34 Vgl. dazu im einzelnen Kutscha, »Europäischer Grundrechtsschutz - in guten Händen? «, in Müller-Heidelberg (Hrsg), Grundrechte-Report 2001, S. 189 ff., der zwischen den Entscheidungen des EuGH zu Diskriminierungen und den Entscheidungen zu Fragen der Effektivität des Gemeinsamen Marktes unterscheidet, bei denen der EuGH stets die Entscheidungen der EG gegenüber individuellen Interessen bestätigt habe.

35 Vgl. Will, Grundrechte der Europ. Union, in Grundrechte-Report 2001, S. 194 ff.; Streinz, EUV/EGV, Kommentar 2003, zu Art. 35 EUV, insbes. Rdnr. 12 ff.; sowie Callies, Kohärenz und Konvergenz beim europäischen Individualrechtsschutz, NJW 2002, 3577.

Zum Fehlen echter Verhältnismäßigkeitsabwägungen bei Eingriffen von EG-Organen in die Freiheitssphäre der Bürger vgl. Caspar, Nationale Grundrechtsgarantien und sekundäres Gemeinschaftsrecht, DÖV 2000, 344, 356 ff. Vgl. demgegenüber die durchaus positive Würdigung der Grundrechtsrechtsprechung des EuGH bei Schwarze, Der Schutz der Grundrechte durch den EuGH, NJW 2005, 3459 ff., der im übrigen dem BVerfG empfiehlt, im Sinne einer besseren Kooperation der Gerichte auch selbst von dem Vorlageverfahren Gebrauch zu machen.

36 Vgl. EuGH, EuGRZ 1995, 316, 319.

37 Vgl. dazu Tettinger, Die Charta der Europäischen Union, NJW 2001, 1010 ff.

38 Vgl. im übrigen auch die kritische Würdigung der Grundrechtsformulierungen bei Alber/ Wittmaier, Mögliche Konfliktbereiche und Divergenzen im europäischen Grundrechtsschutz, EuGRZ 2006, 113 ff., 123.

39 Vgl. »Solange II«: BVerfG Beschluß v. 22.10.1986, NJW 1987, 577 ff.; vgl. »Maastricht«: BVerfG Urteil v. 12.10.1993, NJW 1993, 3047 ff.; vgl. Bananen-Beschluß v. 7.6.2000, NJW 2000, 3124 ff.; vgl. EurHaftbefG Beschluß v. 18.7.2005, NJW 2005, 2289 ff. 
»solange« der EuGH den »als unabdingbar gebotenen« Grundrechtsschutz »generell« in einer »im wesentlichen gleich zu achtenden « Weise gewährleistet. ${ }^{40}$

Aber gerade diese Formeln stehen in Widerspruch dazu, daß die Wahrung der Grundrechte eine individuelle, die jeweilige Rechtsverletzung betreffende und keine statistische Aufgabe ist. Das BVerfG ist kein Gericht im Wartestand, das seine Aufgaben dann wieder übernehmen kann, wenn es mit der Entscheidungspraxis des EuGH generell nicht mehr zufrieden ist. Es kann von einem Beschwerdeführer nicht verlangen, zur Begründung der Zulässigkeit einer Verfassungsbeschwerde erst reihenweise Fehlentscheidungen des EuGH in anderen Sachen vortragen zu müssen, um dann die Verfassungsbeschwerde im eigenen Fall inhaltlich begründen zu dürfen. Das kann auch in Ansehung des Art. 23 GG nicht richtig sein. Wenn das BVerfG in diesen Entscheidungen betont, daß »der Wesensgehalt der Grundrechte und zumal der Menschenrechte unabdingbar ist und auch gegenüber der Hoheitsgewalt der Gemeinschaft Bestand haben « muß und daß es die Aufgabe dieses Schutzes gemeinsam mit dem EuGH in einem Kooperationsverhältnis wahrnehme, dann sollte diese Arbeitsteilung eine präzisere Grundlage haben. ${ }^{41}$

Politisch ist die Rechtsprechung des BVerfG praktisch. Ihre Bewertung hängt davon $\mathrm{ab}$, ob man die Europa-Offenheit des Grundgesetzes als übergeordneten Wert und die ökonomische Nützlichkeit einer Entscheidung der Gemeinschaft als ausreichende Begründung für einen Grundrechtseingriff akzeptiert, oder ob man im Interesse der Grund- und Menschenrechte jedes einzelnen Menschen der Überzeugung ist, daß auch ein Konflikt in Kauf genommen werden muß, wenn es nicht anders geht. ${ }^{42}$

$40 \mathrm{Zu}$ der Frage, wie weit der Grundrechtsschutz durch Art. 23 Abs. 1 GG abgebaut werden könne, vgl. Classen, in Mangoldt/Klein, GG II, 2000, Art. 23, Rdnr. 49 ff. Zum Datenschutz: Zieschang, Der Austausch personenbezogener Daten mittels Europol, ZRP 1996, 427 ff. Dazu gehört aus gutem Grund auch die Frage, ob die Entgegennahme von Daten zulässig ist, die durch Folter erhoben wurden. Nach einer Entscheidung des Rates v. 3.10.1998 - 99/C 26/03, Amtsbl. 1999, C 26 - muß jedenfalls eine Speicherung von Daten durch Europol unterbleiben, wenn »offenkundig ist, daß sie von einem Drittstaat unter offensichtlicher Verletzung der Menschenrechte erhoben wurden.«

41 Vgl. BVerfG »Solange II«, a.a.O., NJW 1987, 582. Maunz-Dürig versteht unter »generell« eine grundsätzliche, wesentliche Grundrechtsfrage. Art. 234 (ex Art. 177) EGV bedürfe der »einschränkenden Auslegung, daß in Fällen einer entsprechend ,generellen' Grundrechtsrelevanz die auslegungsmäßige Vorabentscheidung des EuGH die Kontrollzuständigkeit des BVerfG nicht ausschließen darf.«, vgl. a.a.O, Rdnr. 24 zu Art. 23 GG. Limbach meint, der Grundrechtsschutz könne nach der Absicht des Vertrages hinter dem nationalen Grundrechtsschutz zurückbleiben. In Anbetracht der Vielfalt der Mitgliedsstaaten könne man nicht verlangen, daß die Union und der Gerichtshof den Anforderungen aller nationalen Verfassungen genüge, vgl. Limbach, Die Kooperation in der zukünftigen europäischen Grundrechtsstruktur, Vortrag v. 29.6.2000, EuGRZ 2000, 417 ff. In der Maastricht-Entscheidung, a.a.O., NJW 1993, 3050, geht das BVerfG davon aus, daß die Bereiche Justiz- und Innenpolitik zwar Gegenstand der europäischen Zusammenarbeit der Union, aber bewußt nicht in die supranationalen Zuständigkeiten der EG eingegliedert worden sind. Werde von der Gemeinschaft Hoheitsgewalt ausgeübt, dann müsse sie demokratisch legitimiert sein. Darum sei es entscheidend, daß die demokratischen Grundlagen der Union schritthaltend mit der Integration ausgebaut werden, vgl. a.a.O., S. 3051. 
Gerade angesichts der dargestellten Explosion administrativer grundrechtsrelevanter Entscheidungen im weitgehend parlamentsfreien »Raum der Freiheit, der Sicherheit und des Rechts « ist es dringend notwendig, die Abgrenzung der Zuständigkeit beider Gerichte auf eine rationalere Basis als die der Kooperation zu stellen. Angesichts der Pupino-Entscheidung des $\mathrm{EuGH}^{43}$ wird das BVerfG auf Dauer die Frage nicht umgehen können, was zu gelten hat, wenn nationales Verfassungsrecht und sekundäres Gemeinschaftsrecht miteinander kollidieren, insbesondere dann, wenn das Gemeinschaftsrecht dem nationalen Gesetzgeber keinen Spielraum mehr läßt, den er verfassungsgemäß ausschöpfen könnte und wenn es bei der Rechtsprechung des EuGH bleiben sollte, daß die nationalen Grundrechte bei der Umsetzung von Rahmenbeschlüssen keine Rolle mehr spielen. Dann sollte das BVerfG mit einer Solange III-Entscheidung Farbe bekennen, wie es das in seiner Solange II-Entscheidung auch schon angedeutet hat. ${ }^{44}$

5. Das BVerfG sollte diese Entscheidung nicht fürchten. Dazu sollte es auf seine eingangs erwähnte Rechtsprechung zurückgreifen.

a. Es ist eine originär nur dem Bundesverfassungsgericht zukommende und ausschließlich nach den Normen des Grundgesetzes zu entscheidende Frage, ob eine Änderung der Europäischen Verträge und ggf. die Übertragung weiterer Hoheitsrechte auf die Gemeinschaft formell und materiell ordnungsgemäß und in Übereinstimmung mit den Vorgaben unserer Verfassung zustande gekommen ist.

Dabei muß es prüfen, ob die Staatlichkeit der Bundesrepublik erhalten bleibt, ob die Abgrenzung der übertragenen Rechte und Befugnisse den Voraussetzungen der Bestimmbarkeit genügt, ob die demokratische und rechtsstaatliche Struktur der Union den Voraussetzungen des Art. 23 GG entspricht und ob sie einen im wesentlich gleichen Grundrechtsschutz gewährleistet. Hat das BVerfG diese Voraussetzungen bejaht oder sind die im BVerfGG für eine Anfechtung des Zustimmungsgesetzes vorgesehenen Fristen verstrichen, dann können diese Fragen in weiteren Verfahren nicht mehr erneut aufgeworfen werden. Sie sind entschieden.

42 Vgl. Schmid, Ein enttäuschender Rückzug. Anmerkung zum Bananen-Beschluß des BVerfG, NVwZ, 2001, 249 ff.; Nickel, Zur Zukunft des BVerfG im Zeitalter der Europäisierung, JZ 2001, 625 ff. Das BVerfG geht in seinen Entscheidungen leider nicht auf die teilweise massive Kritik an der Rechtsprechung des EuGH zu den individuellen Grundrechten und auf die äußerst restriktiven Entscheidungen zur unmittelbaren Anrufung des EuGH ein.

$43 \mathrm{Vgl}$. Klink/Proelß, Zur verfassungsgerichtlichen Kontrolldichte bei Umsetzungsakten von Rahmenbeschlüssen der Europäischen Union, DÖV 2006, 469 ff.

44 Vgl. auch Scholz, Wie lange bis »Solange III«?, NJW 1990, 941 ff. Vgl. auch den BVerfGKammerbeschluß v. 12.5.1989 zur Tabakrichtlinie: »Soweit die Richtlinie den Grundrechtsstandard des Gemeinschaftsrechtes verletzten sollte, gewährt der EuGH Rechtsschutz. Wenn auf diesem Wege der vom Grundgesetz als unabdingbar gebotene Grundrechtsstandard nicht verwirklicht werden sollte, kann das BVerfG angerufen werden.«, NJW 1990, 974. Vgl. auch Mangoldt-Dürig, Rdnr. 24 zu Art. 23 GG: Probleme entstünden dann, »wenn ein Rechtsakt des sekundären Gemeinschaftsrechts eine grundrechtliche Gewährleistung berührt, die zwar im GG verankert ist, sich aber in den gemeinsamen Grundrechtsüberlieferungen bzw. in der EMRK nicht wiederfindet. In solchen Fällen muß die grundrechtliche Kontrollkompetenz des BVerfG uneingeschränkt bestehen bleiben.« 
Das mag angesichts der verwaschenen und bewußt spekulativen, eher diplomatischen als rechtlichen Formeln etwa des EVerfVE bedauerlich sein. Aber es liegt sowohl bei dem Gesetzgeber wie bei dem rechtsuchenden Bürger, bei zukünftigen Gemeinschaftsverträgen für größere Klarheit und Bestimmtheit zu sorgen. Man darf gespannt sein, wie das BVerfG über die zum EVerfVE anhängige Verfassungsbeschwerde entscheidet. Es sollte nicht nur die Frage der Bestimmtheit der auf die Union übertragenen Befugnisse entscheiden, sondern sich auch dazu äußern, ob nicht die Grenze zur Staatsbildung überschritten wird und daher die für eine Verfassung erforderliche Legitimation nicht mehr durch eine Ratifikation durch den Bundestag, sondern nur durch eine Entscheidung des Souveräns, also des Volkes erreicht werden kann. Der Bundestag kann im Staat, aber nicht über den Staat entscheiden.

Wenn aber das Gemeinschaftsrecht rechtmäßig zustande gekommen ist, dann kann die weitere oder erneute Zuständigkeit des BVerfG nicht davon abhängig gemacht werden, ob es die Rechtsprechung des EuGH in anderen als in dem vor dem BVerfG anhängigen Rechtsstreit für richtig hält oder nicht.

b. Das BVerfG hat das ständige Recht und die Pflicht, darüber materiell selbst zu entscheiden, ob eine Handlung oder ein Rechtsakt der Gemeinschaft die ihr nach Art. 23 GG übertragenen Rechte überschreitet, also ultra vires, ohne eigene Rechtsgrundlage vorgenommen wird.

Diese Prüfung bezieht sich auch auf die Entscheidungen des EuGH, die mit richterlicher Rechtsfortbildung begründet werden. Dieses verfassungsrechtliche Problem taucht auch im innerstaatlichen Recht auf, in dem das BVerfG die Zulässigkeit richterlicher Rechtsfortbildung mit einer gewissen Großzügigkeit behandelt hat. So kann man die richterliche Bildung von - im EG-Vertrag nicht enthaltenen - Grundrechten durch den EuGH durchaus auf Art. 6 EUV (ex Art. F) und seine Entscheidungen über die unmittelbare Verbindlichkeit von Richtlinien darauf stützen, daß ihre inhaltlich und zeitgerecht angemessene Umsetzung vertragsrechtlich erzwungen werden kann. ${ }^{45}$

Die Rechtsprechung des EuGH über die unmittelbare Geltung von Rahmenbeschlüssen - wie im Fall Pupino - müßte jedoch angesichts des eindeutigen Wortlautes des Art. 34 Abs. 2 Zif. b EUV innerhalb der Bundesrepublik vom BVerfG als eine Entscheidung ultra vires aufgehoben werden. Denn der Bundestag hatte bei der Ratifizierung des Vertrages in einer Entschließung ausdrücklich erklärt, daß es sich bei der Zusammenarbeit im justitiellen und polizeilichen Bereich des Titels IV unverändert um die »Dritte Säule« handeln solle, in der Rechtswirkungen nur zwischen den Mitgliedsstaaten eintreten sollen.

Ebenso müßte eine Entscheidung wie die des Europäischen Gerichts (Zweite erweiterte Kammer) in den Sachen Kadi, Yusuf und Barakaat (T 315/01 bzw. T 306/01)

45 Vgl. dazu di Fabio, Richtlinienkonformität als ranghöchstes Normauslegungsprinzip?, NJW 1990, 947 ff. Er weist darauf hin, daß »Solange II « sich auf eine EG-Verordnung bezieht, der EuGH aber in ständiger Rechtsprechung nicht nur die Vertragspflicht der Mitgliedsstaaten zur Durchführung der Richtlinien, sondern auch die Verpflichtung der Gerichte zur richtlinienkonformen Auslegung des nationalen Rechts betont hat, was innerstaatlich auch zu bestandskräftigen Entscheidungen contra legem geführt hat. Damit würden die Proportionen des gewaltenteilenden Systems zu Lasten der Legislative verschoben. 
vom 21.September.2005 als ultra vires vom BVerfG aufgehoben werden, wenn sie sich auf einen deutschen Staatsangehörigen beziehen würden, der auf Grund einer Entscheidung des Sanktionsausschusses der UN »gelistet«, also der völligen Einfrierung von Einkommen und Vermögen ohne vorherige oder nachträgliche Anhörung auf der Grundlage eines Verdachts unterworfen wurde. Der EuGH ist nicht zuständig für die Auslegung des Völkerrechts, ob eine den Mitgliedsstaat auch unmittelbar treffende Verpflichtung aus einer Entscheidung des UN-Sicherheitsrates gegen jus cogens verstößt.

Das Gericht hat in seinen Entscheidungen den Erlaß eine Verordnung der Kommission zur Durchsetzung der UN-Entscheidung im Rahmen der GASP wegen der Bedeutung des Kampfes gegen den Terrorismus für zulässig gehalten, wobei den Betroffenen weder eine Anhörung noch irgendeine Nachprüfung des Verdachts nachgelassen wurde. Das sei weder gemeinschaftsrechtlich möglich, noch völkerrechtlich zwingend erforderlich.

Eine Rechtsgrundlage im EU/EG-Vertrag für eine Verordnung ist entgegen der Auffassung des Gerichts weder ersichtlich, noch war eine Verordnung überhaupt erforderlich, weil alle Mitglieder der Union gleichzeitig Mitglieder der UN sind und eine Verordnung der Kommission die Rechte und Pflichten eines Mitglieds gegenüber einem Beschluß des UN-Sicherheitsrates nicht verändern kann. ${ }^{46}$

c. In gleicher Weise muß das BVerfG Entscheidungen der Gemeinschaft einschließlich des EuGH darauf prüfen, ob sie deswegen ultra vires - also innerhalb der Bundesrepublik ohne Rechtswirkung - ergangen sind, weil sie gegen den ausdrücklich in Art. 23, 79 Abs. 3 GG jeder Übertragung entzogenen Bereich der Art. 1, 20 GG verstoßen. Das gilt gegenüber allen Rechtsakten der Union, ob es Verordnungen, Richtlinien, Rahmenbeschlüsse, Entscheidungen des EuGH oder was immer sind.

Diese Entscheidung kann nicht dem EuGH obliegen, weil er in diesem Bereich mangels Rechtsübertragung keine Zuständigkeit haben kann. Wenn dem nationalen Gesetzgeber die Verfügung über ein Recht vollkommen entzogen ist, dann kann er es auch nicht dadurch beeinträchtigen, daß er seine Definition und seinen Schutz einem Gericht überläßt, das in einem anderen Rechtssystem entscheidet, dessen Rechtsprechung darum nicht ausschließlich dem Grundgesetz und unseren eigenen Rechtstraditionen verpflichtet ist, sondern auch andere Verfassungen und Rechtsüberlieferungen zu berücksichtigen hat.

Art. 23 Abs. 1 GG setzt der Integrationsoffenheit mit dem ausdrücklichen Vorbehalt des Art. 79 Abs. 3 GG eine absolute Grenze. Dazu gehören nicht nur die allgemeinen institutionellen Vorbehalte der Rechtsstaatlichkeit, der Demokratie und der Subsidiarität, sondern auch ausdrücklich die Ewigkeitsgarantie der Wahrung der Menschenwürde durch Art. 1 Abs. 1 GG, der nach unserem Verfassungsverständnis nicht nur als

46 Die Entscheidung müßte auch wegen Verstoßes gegen Art. 6, 17 EMRK und gegen zwingendes Verfassungsrecht aufgehoben werden. Es widerspricht dem Rechtsstaatsgebot, wenn ein Staat gezwungen wäre, Zwangsmittel gegen einen nachweislich Unschuldigen einzusetzen. Der Vorgang ist im Bundestag anhängig gemacht, aber inhaltlich nicht weiter behandelt worden, vgl. BT-Drs. 16/821 v. 7.3.2006. 
Auslegungsrichtlinie oder Programmsatz gilt, sondern der ein konkretes Grundrecht darstellt, und zwar in der Auslegung, die unserer eigenen rechtshistorischen und kulturellen Grundüberzeugung entspricht.

Dabei kommt es nicht auf die Grundlegung von Dürig oder Herdegen, von Böckenförde oder Stern oder wem auch immer an, sondern auf die Rechtsfolge, daß es nämlich nach der ständigen Rechtsprechung des BVerfG einen unbedingten Raum gibt, in dem der Einzelne mit sich allein ist und auf sein Recht vertrauen kann, frei von jeder Abwägungsüberlegung vom Staat in Ruhe gelassen zu werden. Dieser in der Entscheidung des BVerfG zum »Großen Lauschangriff« im Detail ausgeführte Gedanke ist ebenso wie die auf Art. 1 GG gegründete unbedingte Ablehnung der Abwägung menschlichen Lebens im Urteil zum Luftsicherheitsgesetz in dieser Entschiedenheit bisher in keiner anderen europäischen Gerichtsentscheidung zu finden. Die Bestimmung des konkreten Inhalts dessen, was wir unter Menschenwürde verstehen, kann nicht davon abhängig gemacht werden, was andere Parlamente oder Gerichte oder die ganze Breite der jetzigen oder zukünftigen Mitglieder der EU für mit der Menschenwürde vereinbar halten. ${ }^{47}$

Man kann diesen Zuständigkeiten des BVerfG nicht entgegenhalten, daß damit die Rechtseinheit der Europäischen Union gefährdet werde. Es ist nicht Sache des EuGH, eine Rechtseinheit zu schaffen, die über den Willen des Gesetzgebers hinausgeht.

d. Auch die innerstaatliche Umsetzung von Rahmenbeschlüssen unterliegt der Kontrolle des BVerfG nach den Kriterien des Grundgesetzes. Die Überlegung des BVerfG in der Haftbefehlsentscheidung, ob der Gesetzgeber den Ermessensspielraum des Rahmenbeschlusses ausgeschöpft habe, ist jedenfalls dann irrelevant, wenn es dem Gesetzgeber freisteht, das Gesetz überhaupt nicht zu erlassen, was das BVerfG selbst betont. $^{48}$ Wenn ein Rahmenbeschluß auf der Grundlage der »Dritten Säule « Völkervertragsrecht ist, dann ist er - ähnlich der EMRK - dem Grundgesetz jedenfalls nicht übergeordnet. Das Bundesrecht mag zur Vermeidung einer Kollision anhand der Zielsetzung ausgelegt werden, die im Rahmenbeschluß zum Ausdruck gekommen ist. Aber ob das Bundesgesetz zur Umsetzung dieser Absicht verfassungsgemäß war, entscheidet sich allein an den Normen des Grundgesetzes. ${ }^{49}$ Dementsprechend sollten auch die nationalen Parlamente die vom Rat gefaßten Rahmenbeschlüsse solange nicht akzeptieren, als sie gegen die nationalen Verfassungsordnungen verstoßen. ${ }^{50}$

47 Vgl. die Darstellung der völlig unterschiedlichen Verfassungslagen in den Mitgliedstaaten der Union bei Borowsky, in Meyer (Hrsg), Charta der Grundrechte der EU, Nomos 2006, Rdnr. 2 ff. zu Art. 1 (Art. II - 61 EVerfVE), der dem Begriff der Menschenwürde in der Charta allerdings die gleiche Bedeutung beimißt wie in Art. 1 GG.

48 A.A. v. Unger, »So lange« nicht mehr: Das BVerfG behauptet die normative Freiheit des deutschen Rechts, NVwZ 2005, 1266; Masing, Vorrang des Europarechts bei umsetzungsgebundenen Rechtsakten. NJW 2006, 264 ff., vgl. ferner Tomuschat, Ungereimtes/Zum Urteil des BVerfG zum Europ. Haftbefehl, EuGRZ 2005, 453 ff., vgl. Klink/Proelß, Zur verfassungsgerichtlichen Kontrolldichte bei Umsetzungsakten von Rahmenbeschlüssen der Europäischen Union, DÖV 2006, 469 ff.

49 Vgl. dazu insbes. Klink/Proelß, a.a.O., S. 469 ff., die zutreffend fragen, ob Rahmenbeschlüsse der EU mangels eigener Rechtspersönlichkeit überhaupt zugerechnet werden können und die Beschränkung der HaftbefehlsE des BVerfG auf die verbliebenen Spielräume des nationalen Gesetzgebers kritisieren. 
Für diese Rechtslage ist es ohne Bedeutung, daß die Bundesrepublik die Zuständigkeit des EuGH für die Auslegung und Rechtswirksamkeit der Rahmenbeschlüsse nach Art. 35 EUV im Wege von Vorlageentscheidungen anerkannt und sogar eine Vorlagepflicht für letztinstanzliche Gerichte eingeführt hat. ${ }^{51}$ Denn Streitgegenstand vor dem BVerfG sind nicht die völkerrechtlichen Verpflichtungen der Bundesrepublik oder der Inhalt des Rahmenbeschlusses, sondern die Verfassungsmäßigkeit eines Bundesgesetzes, das durch einen Rahmenbeschluß veranlaßt wurde.

e. Schwieriger zu beurteilen ist eine Frage geringerer Bedeutung, ob nämlich das BVerfG eine unmittelbare Verfassungsbeschwerde wegen der Verletzung eines Grundrechtes annehmen kann, wenn zwar die Zuständigkeit des EuGH gegeben ist, aber einen Vorlagebeschluß voraussetzt. Das müßte mit der Folge zulässig sein, daß das BVerfG die Frage dem EuGH zur Entscheidung vorlegt, wenn es die Beschwerde für begründet hält.

6. Ich erwarte allseitige Kritik für den Gedanken, daß das BVerfG über die Vereinbarkeit Europäischer Entscheidungen mit dem Grundgesetz in einem erheblich größeren Umfang selbst entscheiden kann und muß, als sich das aus seiner bisherigen Rechtsprechung ergibt.

Es kann schon sein, daß dann auch andere Mitglieder der Union entsprechende Vorbehalte des nationalen Verfassungsrechts machen. Aber es hat auch bisher weitreichende Vorbehalte einzelner Mitgliedsstaaten wegen nationaler Interessen gegeben, wenn auch mit anderer Begründung - von Großbritannien, Irland, Dänemark und anderen. Der ganze Artikel 33 EUV beruht auf der Forderung Frankreichs, die nationalen Grenzkontrollen gegenüber Belgien und Luxemburg nach 1996 entsprechend Art. 2 Abs. 2 SDÜ auf unbestimmte Zeit fortführen zu können. ${ }^{52}$

Das mag nicht erfreulich sein. Aber die Union würde daran keineswegs zerbrechen, im Gegenteil. Viele Bürger würden eine Ausdehnung der europäischen Befugnisse leichter hinnehmen, wenn sie sicher sein können, daß das ihnen vertraute Bundesverfassungsgericht eine größere Verantwortung dafür übernehmen würde, daß die dem Grundgesetz entsprechende Abwägung von Freiheit und Sicherheit nicht ständig weiter durch Beschlüsse der Exekutiven verschoben werden kann. Und die Exekutiven würden die Grenzen ihrer Beschlußmöglichkeiten viel exakter prüfen und dabei beachten müssen, daß diese Beschlüsse in enger Übereinstimmung mit den Grundsätzen der Subsidiarität und vor allem mit den verbürgten Freiheitsrechten der Bürger stehen müssen und sich auch vor einem nationalen Gericht zu bewähren haben.

50 Vgl. näher Kaiafa-Ghandi, Bemerkungen zur Entwicklung rechtstaatlicher Grundlagen eines Europol-Strafrechts, KritV 2001, 290 ff., 294.

51 Vgl. EUGH-Ges. v. 6.8.1998, BGB1. 98 I 2035.

52 Das sei ein unantastbarer Kernbereich der nationalen Souveränität, vgl. Hailbronner/ Thiery, Amsterdam - Vergemeinschaftung der Sachbereiche Asyl, Einwanderung, Schengen - Besitzstand, EuR 1998, 583 ff. 


\section{Schlußbemerkung}

Das engere Zusammenwachsen der Europäischen Union ist ein wichtiges politisches Ziel. Es darf nicht aufgegeben werden. Das Scheitern des Vertragsentwurfes einer Europäischen Verfassung aus überwiegend einzelstaatlichen Motiven entspricht aber der politischen Wirklichkeit, daß Wirkungsweise und Mechanismen der Europäischen Gremien den meisten Bürgern nicht bekannt sind, von ihnen nicht verstanden werden und sie darum auch nicht besonders interessieren - »solange « sie von ihren Entscheidungen nicht unmittelbar persönlich berührt werden.

Man kann die Zukunft der Europäischen Gemeinschaft nur dadurch sichern, daß man klare und unzweideutige Ziele setzt, die Bürger an der Verfassungsgesetzgebung beteiligt und sicherstellt, daß die grundlegenden nationalen Verfassungsrechte so lange gesichert und erhalten werden, bis eine europäische Bevölkerung einen Europäischen Staat bilden will, aus politischem Willen und aus politischer Einsicht, und nicht zur Bewältigung verwaltungstechnischer Zwangsläufigkeiten.

Darum kann man die europäische Entwicklung nicht der immer engeren Zusammenarbeit von Fachbeamten, Fachpolitikern und Experten überlassen. Man muß sie auf eine demokratische Grundlage stellen.

Ein neuer Verfassungsvertrag sollte die Zuständigkeiten der Gemeinschaft klar definieren.

Jede verbindliche Gesetzgebung sollte an eine Mitentscheidung des Europäischen Parlamentes gebunden werden.

Der Vertrag sollte in jedem einzelnen Mitgliedsland einer Volksabstimmung unterworfen werden und es sollte dabei der Entscheidung jedes einzelnen souveränen Verfassungsgebers überlassen werden, ob ein Vorbehalt zugunsten der nationalen Verfassungsgerichtsbarkeit im Sinne einer unbedingten Mindestgarantie entsprechend dem Art. 17 EMRK gegenüber allen Entscheidungen der Unionsorgane gemacht wird oder nicht.

Der deutsche Bundestag sollte erheblich mehr unternehmen, um eine demokratische Legitimation der europäischen Entscheidungen zu sichern. Er sollte in das Zusammenarbeitsgesetz einen bindenden Parlamentsvorbehalt für diejenigen Entscheidungen der Exekutive in europäischen Gremien einfügen, die zur Umsetzung ein Gesetz benötigen, zumindest aber, soweit diese Entscheidungen die Verfassung berühren. Leider hat der Bundestag in der am 22. September 2006 beschlossenen Vereinbarung mit der Bundesregierung zu $\S 6$ des Zusammenarbeitsgesetzes einen solchen Parlamentsvorbehalt nicht durchgesetzt.

Und schließlich sollte es die Europäische Union entschlossen unternehmen, der Europäischen Menschenrechtskonvention beizutreten. 